\title{
OLD CONCEPTS, NEW IDEAS: APPROACHES TO TRANSLATION SHIFTS
}

\author{
Lea Cyrus \\ University of Münster
}

\begin{abstract}
This paper traces the development of the translation shift concept from its origins in the linguistics-oriented era of translation studies to its current revival in computer-based approaches: after a presentation of the traditional approaches by John C. Catford, Jean-Paul Vinay and Jean Darbelnet, Eugene A. Nida, and Kitty van LeuvenZwart, several recent studies that have integrated the concept of translation shifts are introduced and discussed. This comparison of old and new approaches reveals that the attitudes towards shifts have changed from mildly prescriptive to neutrally descriptive. The paper concludes with a general evaluation of the place of linguistic approaches in translation studies.
\end{abstract}

\section{Zusammenfassung}

Dieser Artikel untersucht die Entwicklung des Shift-Konzepts von seinen Ursprüngen in der linguistisch orientierten Phase der Übersetzungswissenschaft bis hin zu seiner derzeitigen Neubelebung in computerbasierten Ansätzen. Es werden zunächst die traditionellen Ansätze von John C. Catford, Jean-Paul Vinay und Jean Darbelnet, Eugene A. Nida sowie Kitty van Leuven-Zwart dargestellt und dann einige neuere Untersuchungen, die sich mit dem Shift-Konzept befassen, vorgestellt und diskutiert. Durch diese Gegenüberstellung wird deutlich, dass die präskriptiven Standpunkte mittlerweile einer neutral deskriptiven Herangehensweise gewichen ist. Der Artikel endet mit einer generellen Einschätzung des Stellenwerts linguistischer Ansätze in der Übersetzungswissenschaft.

Keywords: Translation shifts. Linguistic translation studies. Computer-based translation studies. History of translation studies. Equivalence.

Schlagwörter: Translation-Shifts. Linguistische Übersetzungswissenschaft. Computerbasierte Übersetzungswissenschaft. Geschichte der Übersetzungswissenschaft. Äquivalenz 


\section{Introduction}

The discipline of translation studies has come a long way. Until well into the 20th century, discourse on translation largely centered around methodological questions and was mainly conducted by practicing translators to justify their own approaches or to criticise those of others, and it was aimed at the establishment of prescriptive guidelines for future translations (see the various texts collected by Störig 1963).

After WWII, the developments in modern linguistics (first structuralism, later also generative grammar) and the early enthusiastic and optimistic attempts at machine translation fuelled the belief that it was possible to develop precise and objective theories of translation. During this time, translation studies was "clearly defined as a sub-discipline of applied linguistics" (SnellHornby 1990: 80), and it was then that the concept of equivalence (see e.g. Kenny 1998) became a key concept of translation studies. Drawing up correspondences between two linguistic systems was one of the central tasks during this time (see e.g. Kade 1968). However, due to the incommensurability of linguistic systems, actual translations always involve shifts, which "result from attempts to deal with systemic differences" (Bakker et al. 1998: 226), so many theories of translation at the time also included a systematisation of such translation shifts.

In subsequent years, the focus of attention in translation studies moved away from these narrowly linguistic and basically source-oriented approaches, which were accused of prescriptivism and of the "negative kind of reasoning required by any search of shifts, which [...] would encompass all that a translation could have had in common with its source but does not" (Toury 1995: 84). Instead, the focus shifted towards the target culture and towards the function and place that a translation has in it, and Toury's (1995) Descriptive Translation Studies and Beyond and his concept of norms gained enormously in influence. Slowly but surely translation studies developed into an independent academic discipline that emancipated itself from linguistics and today deals with a great variety of questions, including questions of gender or postcolonial power relations (see Snell-Hornby 2006 for a comprehensive account of all the turns the discipline took over the years).

However, translation still involves language - not exclusively so, but undeniably so - and consequently there are still linguistic approaches to translation, even though they tend to be eyed with suspicion by those who still vividly remember the painful process of emancipation (Snell-Hornby [2006: 152] speaks of a "phase of retrogression"). In recent years it is particularly the development of corpus linguistics that has given rise to new linguistic 
investigations into translation, which differ from their predecessors in that they are now empirically oriented and descriptive rather than theoretical and prescriptive. The primary focus of this new paradigm lies in discovering properties of translated language as opposed to non-translated language, i.e. in identifying those "patterns in translation that are not the result of interference from either source or target language" but rather "a product of constraints inherent in the translation process" (Olohan 2004: 92). Currently, it is a topic of heated debate whether these properties of translated language can even be seen as translation universals (Mauranen \& Kujamäki 2004). Research into potential translation universals is normally conducted using so-called comparable corpora, i.e. monolingual corpora that contain non-translated and translated subcomponents which are comparable in terms of genre, register, time of writing, text size, etc. Frequently, the texts in the translational subcorpus have been translated from a variety of different languages, which makes it possible to rule out interference from one particular source language (see e.g. Laviosa 2002).

With the availability of parallel corpora, i.e. bi- or even multilingual corpora that contain source texts alongside their translations into one or several languages, the concept of translation shifts has gradually regained in importance, too. However, the perspective on shifts has changed considerably: they are no longer viewed as "mistranslations" or "deviations of the norm" (van Leuven-Zwart 1990b: 228), neither are they reduced to being a way of coping with systemic differences. Instead, it is now widely accepted that shifts can be caused and influenced by a variety of other factors, too, some of them extralinguistic, such as the function of the translated text in the target culture or a subjective interpretation by the translator (van Leuven-Zwart 1990b: 228). The prescriptive undertone has completely disappeared, and shifts are now recognised as a "phenomenon inherent to translation" (van Leuven-Zwart 1990b: 228) or even "a defining feature of translation" (Toury 2004: 22), which makes them a suitable object of investigation within descriptive translation studies and the empirical corpus-based approach. Furthermore, some recent studies suggest that the concept of shifts can also be usefully employed in certain practical applications, such as in the evaluation of machine translation output, so shifts are also potentially of interest in applied translation studies.

This paper will trace the origins and the development of the shift concept from its beginning in the middle of the 20th century to its new guise in the computer age, in order to show how old concepts can change over time and thus give rise to promising new avenues of research. 


\section{Old Concepts: Origins and Development of the Translation Shift Approach}

\subsection{John C. Catford}

The term "translation shift" was first introduced by Catford (1965). His definition of this concept relies on his distinction between formal correspondence and textual equivalence: formal correspondence is a relationship that holds between two linguistic categories that occupy approximately the same place in the organisation of their respective languages, while textual equivalence holds between two portions of text that are actual translations of each other. When a textual equivalent is not formally correspondent with its source, this is called a translation shift, of which there are two major types: level shifts (section 2.1.1) and category shifts (section 2.1.2).

One of the problems with this approach is that it presupposes that it is actually feasible to determine those elements in two linguistic systems that are formal correspondents of each other - after all, this is a prerequisite for deciding whether there is a divergence between formal correspondence and textual equivalence. For this reason, Catford's account remains purely theoretical and has never been fully applied to any actual translations.

\subsubsection{Level Shifts}

Catford (1965: 73) speaks of a level shift when a source text item has a textual equivalent on a different linguistic level. Following the early Hallidayan Scale and Category Grammar (see Halliday 1961), he distinguishes the four linguistic levels - phonology (the medium-form of spoken language), graphology (the medium-form of written language), grammar (closed systems), and lexis (open sets) -, which are related in language-specific ways to extra-linguistic levels of substance: phonology to phonic substance, graphology to graphic substance, and both grammar and lexis to situation substance.

Level shifts, however, can only occur between the levels of grammar and lexis. This restriction is due to Catford's understanding of translation equivalence, which, from his structuralist point of view, is not based on a sameness of meaning, for meaning is defined as "the total network of relations entered into by any linguistic form" (Catford 1965: 35) and consequently cannot be the same across languages. Rather, the prerequisite for translation equivalence is that two linguistic elements can function in the same situation, and this is only possible if there exists a certain overlap of relevant situational features on the level of substance. Consequently, textual and translational equivalence 
is only possible between elements that relate to the same level of substance, and this is the case only for the linguistic levels of grammar and lexis.

One example of such a level shift would be the translation of an aspectual category - i.e. an item on the level of grammar - with a lexical item that conveys a similarly aspectual meaning, for instance the translation of a Russian perfective aspect with the English lexical verb "to achieve".

\subsubsection{Category Shifts}

There are four types of category shifts: unit, structure, class, and intra-system shifts. Just like the definition and classification of levels, these four categories are also taken over from Halliday (1961).

A unit is defined as "a stretch of language activity which is the carrier of a pattern of a particular kind" (Catford 1965: 5). Units are organised hierarchically on a rank scale, with each unit being composed of a specific pattern of units that are (normally) lower in rank. The grammatical rank scale consists of the units sentence, clause, group, word, and morpheme. Consequently, a unit shift occurs when textual equivalents are located on different ranks, as is e.g. the case when a clause is translated as a group.

A structure is defined as the patterned way in which a unit is made up of lower-rank units. A structure shift thus occurs when the target structure contains different classes of elements or else when it contains the same classes of elements, but arranges them differently. According to Catford (1965: 77), structure shifts are the most frequent among the category shifts. As an example, Catford presents the translation of an English clause consisting of the elements subject, predicate, and complement, into a Gaelic clause that is composed of the elements predicate, subject, complement, and adjunct.

Those types of elements that can perform the same function in the unit next above in rank scale form a class (a different term would be "syntactic category"). An example of a class shift would thus be the translation of English "a medical student" into French "un étudiant en medicine", because the textual equivalent of the English adjective phrase is a French prepositional phrase and thus of a different class.

In Catford's theory, the concept of system has a more restricted meaning that in other structuralist approaches, where it usually refers to the relations that hold within a language in its entirety. Here, the term is used for "a finite set of alternants, among which a choice must be made" (Catford 1965: 7), for example the system of pronouns or of number. An intra-system shift occurs when the two languages have a formally correspondent system, but choose a non-corresponding item as translation equivalents. For instance, English and 
French both have a two-place number system, but the English plural "trousers" is translated as the French singular "le pantalon".

\subsection{Jean-Paul Vinay and Jean Darbelnet}

Even though Catford (1965) is credited with introducing the term "translation shift", he was by no means the first to deal with the phenomenon. Vinay and Darbelnet's (1958) contrastive analysis of English and French is an attempt at providing a systematic survey of the differences that exist between the two languages, with the aim of enabling the translator to avoid the pitfalls associated with this particular language pair. This distinctly didactic orientation distinguishes them from more mainstream structuralist approaches, which tend to be purely theoretical. However, the pedagogical dimension also gives rise to a certain prescriptive undertone.

A central element of their work is a system of seven translation procedures which translators apply when moving from source to target message. The first three of these procedures - borrowing, calque, and literal translation - are classified as direct translations, while the last four of them are oblique. It is these oblique procedures that result in various differences between the source and target text - shifts, in other words, even though Vinay and Darbelnet did not call them that. These oblique procedures are called transposition (section 2.2.1), modulation (section 2.2.2), equivalence (section 2.2.3), and adaptation (section 2.2.4).

\subsubsection{Transposition}

Transposition is a change of word class that does not affect the overall meaning of the message. Theoretically, transposition can happen between all kinds of word classes - Vinay and Darbelnet (1958: 97) provide an extensive list - but particular emphasis is placed on the replacements of verbs with nouns or vice versa, as in the translation of French "dès son lever" with English "as soon as he got up".

\subsubsection{Modulation}

As opposed to meaning-preserving transposition, Vinay and Darbelnet (1958: 51) speak of modulation when the translation procedure results in a change of point of view. Modulation can be compared to the monolingual rhetorical devices metonymy or synechdoche, as the various subprocedures illustrate, as, for instance, "cause for effect" (e.g. "You're quite a stranger" vs. "On ne 
vous voit plus", 237) or "part for the whole" (e.g. "He shut the door in my face" vs. "Il me claqua la porte au nez", 238).

\subsubsection{Equivalence}

While the procedure of modulation still leaves a semantically categorisable link between the source and the target items, the procedure of equivalence leads to a replacement of the entire message by completely different lexical, stylistic, and structural means, as is e.g. necessary for the translation of clichés and proverbs. Similarly, this procedure can be applied when the source text contains an allusion to a literary work or historical event that might be lost to a recipient with a different cultural background. This use of the term "equivalence" is quite different from the way it is normally understood in translation studies (see Kenny 1998), which is why Chesterman (1989: 67) prefers to refer to this procedure as "total syntagmatic change".

\subsubsection{Adaptation}

Vinay and Darbelnet (1958: 52) consider their last procedure, adaptation, to be "la limite extrême de la traduction", but the difference between adaptation and equivalence seems only one of degree. Adaptation is applied when the entire situation described in the source message does not exist in the target culture, which might lead the translator to adapt the situation so that the translation describes a similarly ordinary event as the original.

\subsection{Eugene A. Nida}

Just like Vinay and Darbelnet (1958), Nida (1964) published his book (Toward a Science of Translating) before Catford (1965) provided a proper definition of translation shifts, but they nevertheless play a central role in his approach. His background lies in missionary bible translation, so he had to deal with languages that were significantly different from English. One of his key concepts is that of dynamic equivalence: the translation should be formulated in such a way that it evokes a receptor response that is substantially the same as that of the original receptors. This aim can be achieved by "complete naturalness of expression" (Nida 1964: 159), i.e. the translation should ideally read like an original of the target language. Nida gives this equivalence of receptor response precedence over any equivalence of form, which entails that it is necessary for the translator to apply certain "techniques of adjustment" (Nida 1964: 226) during the translation process. These techniques fall into 
three main categories: additions, subtractions (section 2.3.1), and alterations (section 2.3.2).

\subsubsection{Additions and Subtractions}

Nida speaks of addition whenever the target text contains more linguistic material than its source. However, he stresses that only those additions "may legitimately be incorporated into a translation" (1964: 227) that do not change the semantic content of the message, but rather make information explicit that is implicitly present also in the source text. Thus, translating a passive clause as an active clause or shifting the word class from event noun to verb might make it grammatically necessary to insert certain participants into the target text. Another type of addition would be to replace pronouns with more concrete expressions. This might be necessary if the source language differentiates categories like gender or number, while the target language does not. In this case, the use of a pronoun in the target language could be a source of ambiguity, which can be avoided by giving a more explicit reference. This would make implicit information explicit, but it would ensure a similar receptor response for both source and target message. Another case would be the insertion of classifiers before proper names or borrowed expressions which are familiar to the source receptor but less so for the target receptor, as in "river Jordan". A classifier would thus compensate for the lack of cultural background.

Subtractions are the negative counterparts of additions in that they make explicit information implicit without actually diminishing the semantic content of the message. They involve "structural losses" but "are advisable because of the grammatical or semantic patterns of the receptor language" (1964: 231).

\subsubsection{Alterations}

Alterations are a kind of residual category for those shifts that are neither additions nor subtractions. Among these are for instance adjustments on the level of sounds, which should be made if straightforward transliteration of a proper name would be misleading (Nida gives one example where the direct rendering of "Messiah" would be understood as "death's hand"). Changes of grammatical categories like e.g. number, tense, or voice also fall into this category, as do changes in word class, word order, sentence type, or directness of discourse. Furthermore, semantic changes on the level of individual words caused by a different organisation of the lexicon are also classified as 
alterations, and so are exocentric expressions like e.g. metaphors or proverbs that are replaced by different target expressions with a similar function.

\subsection{Kitty van Leuven-Zwart}

The approach by van Leuven-Zwart $(1989,1990 a)$ differs from the previous three in that it is designed and used for the description of actual translations rather than of the relationship between two linguistic systems. A further difference lies in van Leuven-Zwart's general attitude towards shifts. In the approaches described so far, the overall tendency was to see shifts as inevitable but somewhat undesirable. They were accepted as ways of coping with the systemic differences that exist between any two languages, but at the same time, the need to overcome these systemic differences was generally seen as the only justifiable cause for the occurrence of shifts. That is, there was a noticeably prescriptive claim that a translator should only revert to shifts or oblique procedures or techniques of adjustments if a more literal translation brought about some disadvantages with respect to the well-formedness or naturalness of the target text. Van Leuven-Zwart's view of shifts, however, is more neutral. She does not describe what translators could and should do or not do, but simply observes and describes what they actually have done, which enables her to formulate hypotheses as to "the translator's interpretation of the original text and the strategy adopted during the process of translation" (van Leuven-Zwart 1989: 154), or, to use a concept introduced by Toury (1980: 54f.): hypotheses as to their initial norms.

To this end, she developed a two-part method for describing "integral translations of fictional narrative texts" (1989: 154), which consists of a detailed analysis of shifts on the microstructural level, i.e. within sentences, clauses, and phrases, and a subsequent investigation of their effect on the macrostructural level, i.e. "on the level of the characters, events, time, place and other meaningful components of the text" (van Leuven-Zwart 1989: 155). In the context of this paper, it is the microstructural shift analysis that is most interesting. It consists of four steps: first, the units to be compared - van Leuven-Zwart calls them transemes - must be established. In this approach, these are either predicates together with their arguments, or predicateless adverbials. Then, for each pair of source and target transemes, a common denominator is determined - the architranseme. The third step consists of establishing the relationship between the architranseme and the transemes, which is either synonymic or hyponymic. Finally, the pairs of transemes are classified according to this relationship. If both of them are synonymic with the architranseme, they are also synonymic with each other, so there is no 
shift. Else, they fall into one of three major categories of shifts (modulation, modification, and mutation), each of which comprises several subcategories 37 altogether, which is one of the reasons why the model has sometimes been criticised for its complexity (see e.g. Munday 1998: 3).

\subsubsection{Modulation}

Modulation occurs when the two transemes are in a hyponymic relationship with each other. This is the case if one of them is a synonym of the architranseme, while the other is a hyponym. If the target transeme is the hyponym, the shift is classified as a specification, otherwise it is a generalisation. Both types of modulation can occur on the level of semantics and on the level of stylistics, and each of these subdivisions is again subcategorised, depending on what is the element of disjunction between the two transemes. On the level of semantics, this could be an aspectual, subjective, concrete, or intensive element - an example of the latter would be the translation of English "loved" into Spanish "adoraba" (1989: 161). On the level of stylistics, this could be due to a difference in register (e.g. formal vs. informal), a professional element, a text-specific element (identifying e.g. a letter, joke, or fairy tale), a time element (e.g. a neologism or archaic expression), or a culture-specific element. The latter leads to exotisation if it occurs in the target transeme, for instance if the Spanish "la plaza" is translated into English as "the plaza" (1989: 164): superficially, they are the same words, but since their effect in the two texts will be quite different, this is classified as a shift. The reverse case is called neutralisation.

\subsubsection{Modification}

Modification in many ways resembles modulation: it can occur on the level of semantics and of stylistics, and the respective subcategories are the same in number and type. The main difference is that both transemes are hyponyms of the architranseme, so the relationship between them is one of contrast. Furthermore, besides semantic and stylistic modification, there is also syntactic modification, which is however only recorded if it has an effect on the semantic, stylistic, or pragmatic level. Syntactic-semantic modification (1989: 166f.) occurs whenever there has been a change with respect to a grammatical feature such as tense, person, or number, or with respect to grammatical class or function. When the two transemes have the same informative value but are composed of a different number of elements, van Leuven-Zwart (1989: 167) speaks of syntactic-stylistic modification. She distinguishes 
between explicitation (if the target transeme contains more elements than the source) and implicitation (if the target transeme contains fewer elements). Finally, there is syntactic-pragmatic modification, which happens when there is a change in speech act or in thematic meaning. An example of the first is the translation of an interrogative with an affirmative clause, while the latter might be a change of voice or a reorganisation of the clausal elements, as, for instance, topicalisation (van Leuven-Zwart 1989: 167f.).

\subsubsection{Mutation}

Those shifts where it is not possible to establish a relationship between the two transemes are called mutation. The most obvious case is when there is no corresponding transeme, which happens when a transeme has been added or deleted in the translation process (categorised by van Leuven-Zwart [1989: 169] as addition or deletion, respectively). If there is a corresponding transeme in the other language, but the semantic difference is too big, this is categorised as radical change of meaning. Van Leuven-Zwart (1989: 169) gives the example of English "... and were making for the place", which is translated into Spanish as "... y estaban arreglando el sitio". It is indeed difficult to find a common denominator for "to make for" and "arreglar". The only problem here is that it is not only the verbs, but the entire phrases quoted above that constitute one transeme, so it is possible to formulate an architranseme: "place" (van Leuven-Zwart does so herself). Consequently, one could argue that both transemes are in a hyponymic relationship with the architranseme, because they both contain an additional verb. From this point of view, this shift would have to be classified as modification rather than as mutation. This illustrates a problematic aspect of van Leuven-Zwart's definition of transeme and architranseme, namely their length. A transeme consisting of a predicate and its arguments tends to contain so many elements that the comparison actually takes place between subcomponents of the transemes, which may exhibit different and even conflicting types of shifts.

\section{New Ideas: the Translation Shift Approach Today}

It was mentioned in section 1 that the preponderantly linguistic approaches to translation were effectively supplanted by the culturally oriented branch of descriptive translation studies, which still continues to be one of the most prominent areas of research in translation studies today. However, in recent years, one can observe a renaissance of linguistic approaches, certainly encouraged by Baker's (1995) influential suggestions as to how linguistic 
corpora could be applied to the study of translation. In this section, some of these new approaches will be presented to show how the old - and often thought dated - concept of translation shifts is revived and applied to new methods and questions.

\subsection{Corpus-Assisted Analysis of Shifts}

Munday's (1998) computational analysis of a Latin American short story and its English translation is a first attempt at applying basic corpus-linguistic and lexicographical methods to the study of parallel texts, by which he hopes to reduce the arduousness of manual investigations (as performed by van Leuven-Zwart 1989) while increasing the objectivity of the results.

His first step is to investigate the general texture of the two texts by collecting basic word statistics. For instance, he compiles frequency lists for both types and tokens. A comparison of the absolute numbers of tokens reveals that the translation is longer than its source, which can be interpreted as an indication of its greater explicitness. However, Munday shows that such a cursory analysis can be misleading: a second token count, in which all personal pronouns are ignored, gives the opposite result. This can plausibly be explained by the fact that Spanish is a pro-drop language, so the translator had to introduce a number of subject pronouns into the target text. While one could argue that this is indeed some kind of explicitation, it is simply caused by systemic differences between the languages.

Similarly, calculating the type-token ratio is usually a way of calculating the lexical complexity of a text, and comparing this ratio for the source and the target text could be a way to find out whether the translation involves simplification. But when comparing these ratios not for texts of the same language but of two different languages, one must, again, take systemic differences into account: Munday (1998: 4) points out that a language that inflects its adjectives for number and gender (like Spanish) is likely to have a larger ratio than a language that does not (like English), simply because it will have three types as opposed to one for each adjective.

Munday then shows how KWIC concordances ("key word in context") can be combined with a sentence alignment tool to detect shifts in lexical cohesion, which is an important aspect in literary texts. By extracting a concordance for a specific source item and then looking at the translations of this item in the aligned sentences, it is possible to investigate whether it has been translated consistently across the text. Of course, it is not possible to decide automatically whether an inconsistent translation is again due to systemic differences (for instance, it would not be possible to translate Spanish "casa" 
"consistently" into English, where one must differentiate between "house" and "home"), but all in all the computerised approach makes it more feasible to go beyond the individual shift and "see if it becomes a trend over the whole text" (Munday 1998: 10).

In his last - admittedly tentative - experiment, Munday examines adjuncts that have been shifted from sentence-initial to sentence-final position or vice versa. His idea is to use a large control corpus like the BNC to find out whether the position chosen by the translator coincides with the most frequent position of this type of adjunct, which he would interpret as a target orientation, or whether it does not, which he sees as reflecting the translator's idiolect. This conclusion is, however, a little hasty. For one thing, before ascribing the choice of a marked position to the translator's idiolect, one would have to check whether the position of the adjunct in the original is marked, too, in which case this choice might simply result from an attempt to create a similar effect in the translation. For another, Munday's control corpus is not analysed for grammatical functions, so he searches for four very specific adjuncts (such as "in the foyer"), which have a rather low frequency even in large corpora, so it is possibly a little bold to draw conclusions as to the general norm from these occurrences. Also, one can probably not infer much about the norms influencing the translator on the basis of four individual decisions. This is particularly true in this case, where the results were slightly different for each of the adjuncts under investigation, because it is rather unlikely that a translator follows different norms depending on the specific adjunct he or she is translating.

\subsection{Measuring Freeness}

Macken (2007) uses the shift approach to investigate current norms with respect to different degrees of freeness in the translation of different text types, which will influence the way an automatic word-alignment tool will work on these texts. She compiled a 33,000-token corpus of English originals and their translations into Dutch that contains three different text types: computer manuals, press releases and proceedings of plenary debates. These texts were then hand-aligned by annotators who were instructed "to indicate the minimal language unit in the source text that corresponds to an equivalent in the target text" (\$3.1), i.e. to select as few tokens as possible, but as many tokens as necessary to ensure or preserve a two-way equivalence. The result is an extended word-alignment that also allows for different types of multi-word expressions to be aligned as single units. In this model, there are three types of alignment links: regular links for straightforward correspondences, fuzzy 
links to capture various kinds of translation shifts, and null links to be used in cases where a portion of text has been added or deleted and thus cannot be aligned. Additionally, null links are used when the translation involves an amount of paraphrase that makes it difficult or impossible to identify corresponding text units.

Macken's study reveals that the three text types do indeed differ with respect to the degree of literalness, which suggests that different norms have been applied in their translation: the source and target computer manuals are the closest, with $92 \%$ of regular links, while the translations of the plenary proceedings are comparatively free with only $81.6 \%$ of regular links (\$4). The press releases range in between, but are a little closer to the literal end of the spectre with $89.3 \%$ of regular links (§4).

Although these results are significant enough to be considered a satisfactory result for what Macken set out to investigate, they are nevertheless problematic. If one compares Macken's results with those of van Leuven-Zwart (1990a), the comparatively large number of regular links across the three text types is striking, for van Leuven-Zwart detected shifts in the majority of her transemes. One possible explanation of this divergence may be that van Leuven-Zwart investigated yet another text type - fictional prose - and that this text type is generally translated much more freely than those investigated by Macken. However, since Macken gives little or no indication of how different two corresponding units must be for them to be classified as fuzzy rather than regular, nor of how different they must be so as to be assigned a null rather than a fuzzy link, it is difficult to assess whether the two studies are at all comparable.

Furthermore, it is questionable whether this rather coarse way of classifying shifts is actually suitable for measuring the freeness of translations, in particular if much of the annotation is restricted to the level of words. For instance, one might take a large number of null links to be an unmistakable sign of a great degree of freeness. However, many of these null links may simply be due to grammatical differences between the two language and thus mark the presence or absence of prepositions, determiners or the like, which would not indicate major shifts at all but merely reflect the different grammatical requirements. While the idea to use shifts as indicators of freeness in translations is plausible, the results might be more reliable if more care were taken to distinguish between different types of shifts. 


\subsection{Shift Annotation}

Cyrus (2006) introduces a small-scale manual annotation project that focuses explicitly on the annotation and categorisation of translation shifts. What distinguishes this project from similar ones is that the alignment and shift annotation is based on predicate-argument structures. The rationale behind this is that such structures are generally seen as representing the underlying meaning of clauses and sentences: two sentences may be realised differently, but if they have a common predicate-argument structure, they can be said to express approximately the same meaning, like e.g. corresponding active and passive sentences. Since predicate-argument structures have proven useful for describing the intralingual relationships between sentences, it seems a plausible step to use them for describing interlingual relationships, too: sentences that are translations of each other should ideally express more or less the same meaning and can consequently be expected to have similar predicateargument structures.

At first sight, basing the shift annotation on predicate-argument structures seems like a direct adaptation of van Leuven-Zwart's transemes (see section 2.4). However, one of the problems with her transemes was that they were too long, because they comprised predicates together with their arguments. In Cyrus' approach, however, each predicate and each of its arguments represents a transeme in itself, i.e. there are predicate transemes and argument transemes, which can be assigned shifts separately.

The texts in her corpus are English originals and their German translations taken from the Europarl corpus (Koehn 2002), which contains proceedings of the European Parliament. In a first step, the monolingual subcorpora are annotated for predicate-argument structure. Since this annotation is a means to an end, it is kept deliberately simple, i.e. no attempt is made at generalising the argument roles along the lines of semantic cases. Arguments are given short, intuitive role names that are predicate-specific (e.g. entity_inviting or entity_invited). The only generalisation is that arguments are used consistently within a predicate group, which consists of related predicates, for instance a verb and its nominalisation.

In a second step, the source predicates and source arguments are aligned to their target counterparts. When the correspondence is straightforward, the alignment is simple, but whenever there is a shift, the alignment is tagged with an appropriate shift tag. The shifts are categorised according to whether they occur on the level of grammar, semantics, or structure. Grammatical shifts are comparatively uncomplicated. They comprise changes in grammatical category, (de)passivisation, (de)pronominalisation, or changes in number 
or definiteness. Among the semantic shifts, there are explicitation and generalisation, which are used when the target transeme is lexically more or less specific than its source, or when some information has been added to or deleted from it. This is distinguished from addition and deletion - these tags are only used when an entire transeme has been added or deleted. When there is some kind of a semantic divergence between two predicates or arguments, this is tagged semantic modification, and mutation is chosen when it is possible to tell that two transemes are textual equivalents, maybe because they have similar arguments, even though they differ radically in meaning (these two types of shifts bear great resemblance to their namesakes in van LeuvenZwart 1989).

Shifts on the level of structure are the most complex changes. Examples here are cases where a transeme is not realised as part of a predicate-argument structure in the other language, but as some kind of adjunct (like the translation of the full lexical verb "to wish" as the adverb "gern", "gladly"). It would not be justified to speak of addition or deletion here, so those adjuncts that correspond to a transeme are annotated too, and the alignment receives a special tag. Another instance of structure shifts occurs when one predicate is split into two different predicates or into a predicate in combination with one of its arguments.

\subsection{Evaluation of Machine Translation}

Ahrenberg and Merkel (2000) propose a correspondence model based on structural and semantic shifts, which can be used for the linguistic evaluation of machine translation output. Their main idea is that the type and amount of structural and semantic divergence that exists between an original and its translation is a text-specific and norm-governed property that should be included in the evaluation of machine translation output, which is currently only evaluated with respect to accuracy.

They chose four different combinations of text type and translation method (computer manuals translated by human translators, computer manuals translated with a translation memory, fictional prose translated by a human translator, and very restricted domain-specific dialogue translated automatically by machine). For each combination, 100 sentence pairs were manually annotated for semantic and structural shifts.

The structural shifts are subdivided into simple and complex shifts. Simple shifts include changes related to the function and properties of clauses (e.g. shifts in voice or mood), to the function and position of constituents (e.g. shifts in grammatical function), and to the number of constituents (e.g. 
additions or deletions). Complex shifts affect several constituents, but cannot be split up into smaller ones. For each pair of sentences, these shifts are added up, which results in a classification of each pair into one of three categories: the sentences are either isomorphic (no structural shifts at all), semi-isomorphic (very few simple shifts), or heteromorphic (a complex shift or several simple shifts).

Then, each pair of content words or multi-unit terms is analysed for semantic shifts: here, the distinction is "more specific", "less specific", or "different meaning". Again, these shifts are added up for each sentence pair, which results in a second classification: the translation is either "equal" (no semantic shifts), "more specific" (exclusively shifts towards the more specific), "less specific" (exclusively shifts towards the less specific), or "other" (shift towards a different meaning, or else a combination of shifts towards the more or the less specific).

With each sentence pair being categorised into one of the structural and one of the semantic classes, it is now possible to calculate for each sample a structural change index for the degree of structural shifts, a semantic equivalence index which measures the proportion of target sentences that have the same meaning as their source, and a specification index that reveals a tendency within the sample towards either specification or generalisation.

Ahrenberg and Merkel suggest to apply this correspondence model to high quality translations of a given text type, to calculate the indexes in the way just described, and then to set the values as the norm for this particular text type. One could then test the norm conformity of a machine translation system by applying the model also to its output and by subsequently comparing the values of the indexes to those of the norm.

What makes this approach interesting is that it acknowledges the fact that shifts are "parts and parcel of high-quality translations and must not be mistaken for errors" (Ahrenberg and Merkel 2000: §5). The authors themselves admit that its obvious drawback is the amount of human effort that is needed for this type of annotation, but they express their hope that, eventually, partial automation will be possible.

\section{Conclusion}

This paper has traced the development of the concept of translation shifts from its origins in the mid-twentieth century to its recent revival in the computer era. While the general idea has remained the same - to deal with divergences between a source and a target text and to develop a classification 
system for them - the attitudes towards shifts and the general orientation of the approaches have changed significantly.

The early proponents had very different backgrounds - ranging from a didactic perspective (Vinay and Darbelnet 1958) to missionary bible translation (Nida 1964) to purely theoretical paradigms (Catford 1965) - but they all had in common that they concentrated predominantly on the relationship between two languages and cultures rather than on the relationship between two actual texts. They all agreed that shifts cannot be avoided when transferring a message from one language to another and are indeed necessary to create a functionally equivalent and natural translation, but there tended to be a prescriptive undertone that a translator should only revert to shifts in cases where a closer translation would yield an unnatural result.

Van Leuven-Zwart's (1989) approach, even though generally counted among the traditional ones, marks a transition towards a more neutral view of the phenomenon and a basically descriptive approach, which today has successfully replaced its theoretical and prescriptive precursors. But it needed the advent of the computer to bring the concept of shifts back to the attention of researchers. Not only did the computer make it possible to overcome some of the drawbacks of van Leuven-Zwart's pen-and-paper model, especially the marked difficulty of applying a complex model consistently across longer texts and of keeping track of the various shifts, it also opened up new areas of application in the fields of corpus and computational linguistics. It remains interesting to see where these attempts at making new uses of old concepts will lead.

Maybe it is time to give up regarding linguistic approaches with suspicion. Translation studies is by now firmly established as an independent academic discipline, and this status will certainly not be called into question by studies that focus on the linguistic aspects of translation. The emancipation from linguistics was definitely an important step, and the discipline is certainly richer for it. However, there comes a stage in every emancipation process when one is so mature that one feels safe to pause and look back to where one has come from - and often this will lead to the insight that all was not bad.

\section{References}

AHREnBERG, Lars \& Magnus Merkel. (2000) "Correspondence Measures for MT Evaluation." In: Proceedings of the Second International Conference on Linguistic Resources and Evaluation (LREC-2000). Athens, Greece. pp. 1255-1261.

BAKER, Mona. (1995) "Corpora in Translation Studies: An Overview and Some Suggestions for Future Research.” Target 7:1. pp. 223-243. 
BAKKER, Matthijs; Cees Koster \& Kitty van Leuven-Zwart. (1998) "Shifts of Translation." In: Baker, Mona (ed.) 1998. Routledge Encyclopedia of Translation Studies. London: Routledge. pp. 226-231.

CATFORD, John C. (1965) A Linguistic Theory of Translation: An Essay in Applied Linguistics. Oxford: Oxford University Press.

Chesterman, Andrew (ed.). (1989) Readings in Translation Theory. Helsinki: Oy Finn Lecture Ab.

Cyrus, Lea. (2006) "Building a Resource for Studying Translation Shifts." In: Proceedings of the Fifth International Conference on Linguistic Resources and Evaluation (LREC-2006). Genoa, Italy. pp. 1240-1245.

HALlidAY, Michael Alexander Kirkwood. (1961) "Categories of the Theory of Grammar." Word 17:3. pp. 241-292.

KADE, Otto. (1968) Zufall und Gesetzmäßigkeit in der Übersetzung. Beihefte zur Zeitschrift Fremdsprachen. Leipzig: VEB Verlag Enzyklopädie.

KENNY, Dorothy. (1998) "Equivalence." In: Baker, Mona (ed.) 1998. Routledge Encyclopedia of Translation Studies. London: Routledge. pp. 77-80.

KoEHN, Philipp. (2002) Europarl: A Multilingual Corpus for Evaluation of Machine Translation. Unpublished draft at http://people.csail.mit.edu/people/koehn/ publications/europarl/.

LaviosA, Sara. (2002) Corpus-based Translation Studies: Theory, Findings, Applications. Amsterdam, Rodopi.

VAN LEUVEN-ZWART, Kitty M. (1989) "Translation and Original: Similarities and Dissimilarities, I." Target 1:2. pp. 151-181.

VAN LEUVEN-ZWART, Kitty M. (1990a) "Translation and Original: Similarities and Dissimilarities, II." Target 2:1. pp. 69-95.

VAN LeUVEn-ZWART, Kitty M. (1990b) "Shifts of Meaning in Translation: Do's or Don'ts?" In: Thelen, Marcel \& Barbara Lewandowska-Tomaszczyk (eds.) 1990. Translation and Meaning, Part 1. Maastricht: Euroterm. pp. 226-233.

MACKEN, Lieve. (2007) "Analysis of Translational Correspondence in View of Subsentential Alignment." In: Proceedings of the METIS-II Workshop on New Approaches to Machine Translation. Leuven, Belgium.

MaUranen, Anna \& Pekka Kujamäki (eds.) (2004) Translation Universals: Do they Exist? Amsterdam, Benjamins.

MundAY, Jeremy. (1998) "A Computer-Assisted Approach to the Analysis of Translation Shifts.” Meta 43:4. pp. 142-156.

NidA, Eugene Albert. (1964) Toward a Science of Translation. Leiden: Brill.

OlOHAN, Maeve. (2004) Introducing Corpora in Translation Studies. London, Routledge.

SnELL-Hornby, Mary. (1990) "Linguistic Transcoding or Cultural Transfer? A Critique of Translation Theory in Germany.” In: Bassnett, Susan \& André 
Lefevere (eds.) 1990. Translation, History and Culture. London: Pinter. pp. 79-86.

Snell-Hornby, Mary. (2006) The Turns of Translation Studies. Amsterdam: Benjamins.

STÖRIG, Hans Joachim (ed.) (1963) Das Problem des Übersetzens. Darmstadt: Wissenschaftliche Buchgesellschaft.

TOURY, Gideon. (1980) In Search of a Theory of Translation. Tel Aviv: Porter Institute.

TOURY, Gideon. (1995) Descriptive Translation Studies and Beyond. Amsterdam: Benjamins.

TOURY, Gideon. (2004) "Probabilistic Explanations in Translation Studies: Welcome as they are, would they Qualify as Universals?" In: Mauranen, Anna \& Pekka Kujamäki (eds.) 2004. pp. 15-32.

VInAY, Jean-Paul \& Jean Darbelnet. (1958) Stylistique Comparée du Français et de l'Anglais: Méthode de Traduction. Paris: Didier. 